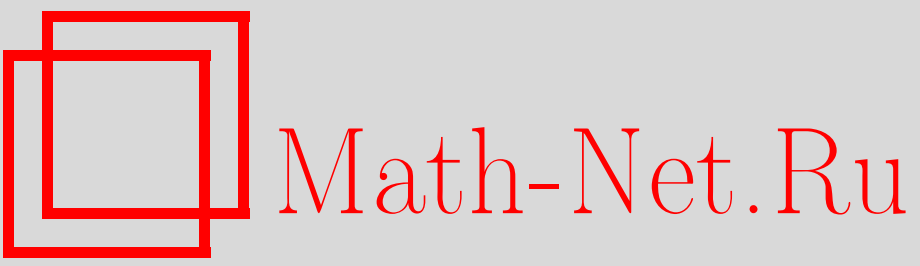

Л. Д. Пустыльников, Об одном свойстве классической дзета-функции, связанном с гипотезой Римана о нулях, УМН, 1999, том 54, выпуск 1, 259-260

DOI: https://doi.org/10.4213/rm128

Использование Общероссийского математического портала Math-Net.Ru подразумевает, что вы прочитали и согласны с пользовательским соглашением

http://www.mathnet.ru/rus/agreement

Параметры загрузки:

IP: 54.89 .56 .158

26 апреля 2023 г., 13:55:17 


\section{ОБ ОДНОМ СВОЙСТВЕ КЛАССИЧЕСКОЙ ДЗЕТА-ФУНКЦИИ, СВЯЗАННОМ С ГИПОТЕЗОЙ РИМАНА О НУЛЯХ}

\section{Л. Д. Пустыльников}

В настоящей работе обнаружено представляющееся важным в связи с гипотезой Римана о нулях (теорема 2) свойство хорошо известной функции $\xi(s)$, полученной из дзета-функции Римана $\zeta(s)$ с помощью равенства $\xi(s)=\frac{1}{2} s(s-1) \pi^{-\frac{s}{2}} \Gamma\left(\frac{s}{2}\right) \zeta(s)$, где $\Gamma(s)$ - гамма функция Эйлера. А именно, здесь доказана следующая

Теорема 1. Все четные производные функции $\xi(s)$ в точке $s=\frac{1}{2}$ строго положиmельнье.

ЗАмечание. В силу равенства $\xi(s)=\xi(1-s)$ все нечетные производные функции $\xi(s)$ в точке $s=\frac{1}{2}$ равны нулю.

ДОКАЗАТЕЛЬСТВо теоремы 1.

ЛЕмма. Пусть $n$ - произвольное натуральное число. Тогда при любом натуральном $r \geqslant 3$ выполнено неравенство

$$
\int_{1}^{\infty}\left(16 r(r-1) \ln ^{r-2} x-\ln ^{r} x\right) x^{-\frac{3}{4}} e^{-\pi n^{2} x} d x>0 .
$$

ДокАЗАТЕльСтво. Введем числа $x_{r}=e^{4 \sqrt{r(r-1)}}, \tilde{x}_{r}=\sqrt{x_{r}}, x_{r}^{*}=e^{r}$ и докажем, что

$$
12 r(r-1) \int_{x_{r-2}^{*}}^{\tilde{x}_{r}}\left(\ln ^{r-2} x\right) x^{-\frac{3}{4}} e^{-\pi n^{2} x} d x>\int_{\tilde{x}_{r}}^{\infty}\left(\ln ^{r} x\right) x^{-\frac{3}{4}} e^{-\pi n^{2} x} d x
$$

В области $x \geqslant 1$ функция $\frac{\ln x}{x^{1 / r}}$ имеет единственный экстремум в точке $x=x_{r}^{*}$, являющийся максимумом, и поэтому в силу неравенства $\tilde{x}_{r}>x_{r}^{*}$ при $x \geqslant \tilde{x}_{r}$ справедливо неравенство $\ln ^{r} x<$ $\frac{\ln ^{r} \tilde{x}_{r}}{e^{\tilde{x}_{r}}} e^{x}$, из которого получаем неравенство

$$
x^{-\frac{3}{4}} e^{-\pi n^{2} x} \ln ^{r} x \leqslant A(x) \stackrel{\text { def }}{=} \frac{\ln ^{r} \tilde{x}_{r}}{e^{\tilde{x}_{r}} \tilde{x}^{\frac{3}{4}}} e^{-\left(\pi n^{2}-1\right) x} \quad\left(x \geqslant \tilde{x}_{r}\right) .
$$

Точно также в области $x_{r-2}^{*} \leqslant x \leqslant \tilde{x}_{r}$ справедливо неравенство

$$
x^{-\frac{3}{4}} e^{-\pi n^{2} x} \ln ^{r-2} x \geqslant B(x) \stackrel{\text { def }}{=} \frac{\ln ^{r-2} \tilde{x}_{r}}{e^{\tilde{x}_{r}} \tilde{x}^{\frac{3}{4}}} e^{-\left(\pi n^{2}-1\right) x} .
$$

Далее, в силу определений $\tilde{x}_{r}$ и $x_{r}^{*}$ получаем:

$$
\frac{3}{4} \int_{x_{r-2}^{*}}^{\tilde{x}_{r}} e^{-\left(\pi n^{2}-1\right) x} d x>\int_{\tilde{x}_{r}}^{\infty} e^{-\left(\pi n^{2}-1\right) x} d x .
$$

Работа выполнена при частичной поддержке Российского фонда фундаментальных исследований (грант № 97-01-00268). 
Поэтому согласно определению чисел $x_{r}, \tilde{x}_{r}$ и соотношению $\frac{A(x)}{B(x)}=\ln ^{2} \tilde{x}_{r}<16 r(r-1)$ из $(2),(3)$ и (4) следует неравенство (1). Заметим теперь, что в области $x_{r-2}^{*} \leqslant x \leqslant \tilde{x}_{r}$ справедливо неравенство $\ln ^{2} x \leqslant 4 r(r-1)$, и поэтому

$$
\begin{aligned}
& 16 r(r-1) \int_{x_{r-2}^{*}}^{\tilde{x}_{r}}\left(\ln ^{r-2} x\right) x^{-\frac{3}{4}} e^{-\pi n^{2} x}-\int_{x_{r-2}^{*}}^{\tilde{x}_{r}}\left(\ln ^{r} x\right) x^{-\frac{3}{4}} e^{-\pi n^{2} x} d x \\
& \quad \geqslant 12 r(r-1) \int_{x_{r-2}^{*}}^{\tilde{x}_{r}}\left(\ln ^{r-2} x\right) x^{-\frac{3}{4}} e^{-\pi n^{2} x} d x .
\end{aligned}
$$

Из этого неравенства и неравенства (1) очевидным образом следует лемма.

Введем функцию $\omega(x)=\sum_{n=1}^{\infty} e^{-\pi n^{2} x}$ и, используя интегральное представление для функции $\pi^{-\frac{s}{2}} \Gamma\left(\frac{s}{2}\right) \zeta(s)$ (см. [1], [2]), получим для любого четного $r \geqslant 4$ равенство

$$
\frac{d^{r} \xi}{d s^{r}}\left(\frac{1}{2}\right)=2^{-(r+2)} \int_{1}^{\infty}\left(16 r(r-1) \ln ^{r-2} x-\ln ^{r} x\right) x^{-\frac{3}{4}} \omega(x) d x .
$$

Из этого равенства и леммы при $r \neq 2$ очевидно следует утверждение теоремы 1 . Что жекасается второй производной функции $\xi(s)$ в точке $s=\frac{1}{2}$, то непосредственная проверка показывает, что она положительная и приближенно равна величине 0,02997 . Теорема 1 доказана.

Теорема 2. Если хотя бъ одна четная производная функции $\xi(s)$ в точке $s=\frac{1}{2}$ бълла бъ не положительная, то гипотеза Римана о нулях функции $\zeta(s)$ была бы неверна: в этом случае существует комплексный нуль функции $\zeta(s)$, не лежащий на прямой $\operatorname{Re} s=\frac{1}{2}$.

ДокАЗАтЕльство. Рассмотрим функцию $\Xi(t)=\xi\left(\frac{1}{2}+i t\right)$ комплексного переменного $t$, где $i$ - мнимая единица. Известно [3], что $\Xi(t)$ - четная целая функция первого порядка, принимающая вещественные значения при вещественных значениях $t$, и любой невещественный нуль $z$ функци $\zeta(s)$ имеет вид $z=\frac{1}{2}+i \rho$, где $\rho-$ любой нуль функции $\Xi(t)$. Поэтому из формулы Вейерштрасса для целых функций [1], [2] следует, что функцию $\Xi(t)$ можно представить в виде

$$
\Xi(t)=e^{a+b t} \prod_{n=1}^{\infty}\left(1-\frac{t}{\rho_{n}}\right) e^{\frac{t}{\rho_{n}}},
$$

где $a$ и $b$ - константы, а числа $\rho_{n}(n=1,2, \ldots)$ пробегают все нули функции $\Xi(t)$. Так как для каждого нуля $\rho_{n}$ среди нулей функции $\Xi(t)$ содержится нуль $-\rho_{n}$, то, предполагая, что гипотеза Римана верна, в силу (5) и четности $\Xi(t)$ имеем равенство

$$
\Xi(t)=e^{a} \prod_{n=1}^{\infty}\left(1-\frac{t}{\tilde{\rho}_{n}^{2}}\right),
$$

где произведение распространено на все положительные нули $\tilde{\rho}_{n}$ функции $\Xi(t)$. Если теперь разложить функцию (6) в ряд Тейлора $\Xi(t)=\sum_{k=0}^{\infty} c_{k} t^{2 k}$ в точке $t=0$, то из равенства (6) будет следовать, что при всех $k$ соседние коэффициенты $c_{k}$ и $c_{k+1}$ имеют противоположные знаки, а это согласно определению $\Xi(t)$ означает, что все четные производные функции $\xi(s)$ в точке $s=\frac{1}{2}$ имеют тот же знак, что и величина $\xi\left(\frac{1}{2}\right)$. Поэтому утверждение теоремы 2 вытекает из неравенства $\xi\left(\frac{1}{2}\right)>0$.

\section{СПИСОК ЛИТЕРАТУРЫ}

[1] Karatsuba A. A., Voronin S. M. The Riemann Zeta-Function. Berlin: de Gruyter, 1992. [2] Карацуба А. А. Основы аналитической теории чисел. М.: Наука, 1975. [3] Титчмарш Е. К. Теория дзета-функции Римана. М.: ИЛ, 1953. 